\title{
Aus gegebenem Anlass: Beendet die Gruppentherapie ihr Schattendasein in der ambulanten Versorgung?
}

\author{
Ulrich Voderholzer
}

Schön Klinik Roseneck, Prien am Chiemsee, Deutschland

Gemäß Zielke [2010] stellt «die Differenziertheit der Gruppenangebote ... ein Qualitätsmerkmal verhaltenstherapeutischer Praxen» dar. Eine provokante Aussage! Tatsächlich wurde diese Einschätzung eben auch falsch zitiert! Korrekt zitiert muss es «Kliniken» statt «Praxen» heißen. Aber was würde es denn für die ambulante psychotherapeutische Versorgungslandschaft bedeuten, wenn auch das falsche Zitat ein Stück weit zuträfe?

Das hieße, dass ambulante Patienten auch in Gruppen behandelt würden und dabei eventuell sogar in den Genuss eines störungs- und/oder problemspezifischen Gruppenangebots kämen. Patienten, die Opfer eines sexuellen Missbrauchs wurden, könnten sich dann untereinander austauschen, und Patienten, die unter einer sozialen Phobie leiden, könnten sich dann nicht nur gemeinsam auf Expositionen vorbereiten, sondern diese auch zusammen durchführen. Diese 2 als Beispiele angeführten Therapieangebote, die zu den am besten bzw. hilfreichsten Gruppenangeboten einer Klinik gehören, würden dann auch ambulanten behandelten Patienten zur Verfügung stehen.

Die Versorgungsrealität ist jedoch eine andere. Obwohl immerhin rund 27\% aller niedergelassenen Therapeuten über eine Zulassung für die Abrechnung von Gruppentherapie verfügen, bieten nur etwa 7\% auch Gruppen an. Die Zahlen korrespondieren mit den Angaben der Krankenkassen, denen zufolge lediglich 1\% der ambulanten Psychotherapien im Gruppenformat durchgeführt werden.

Obwohl Gruppentherapien kostengünstiger sind als Einzeltherapien und durch Gruppen mehr Patienten versorgt werden könnten als es Einzeltherapien ermöglichen, spielen Gruppentherapien in der ambulanten Versorgung praktisch keine Rolle. Während einzeltherapeutische Angebote sozusagen auf der Sonnenseite angesiedelt sind (man denke nur an die große Zahl von reinen Privatpraxen in Großstädten), führen Gruppentherapien in der ambulanten Versorgung ein Schattendasein.

Woher rührt dieser Umstand? Verglichen mit der Durchführung von Einzeltherapien geht die Durchführung von Gruppentherapien mit einem deutlichen organisatorischen Mehraufwand für die Therapeuten einher. Dies betrifft unter anderem die Antragstellung für die Genehmigung der therapeutischen Leistungen, die Zusammenstellung der Gruppe, den Umgang mit Absagen und Therapieabbrüchen sowie das Vorhandensein geeigneter Räumlichkeiten, um nur einige Punkte zu nennen. Dieser organisatorische Mehraufwand relativiert den monetären Anreiz, der sich daraus ergibt, dass gruppentherapeutische Sitzungen besser vergütet werden als einzeltherapeutische. Ein weiterer Grund liegt darin, dass in den meisten Curricula verhaltenstherapeutischer Ausbildungsinstitute gruppentherapeutische Verfahren nur eine marginale Rolle spielen und deshalb durchaus Kompetenzdefizite in Bezug auf die die Leitung von Gruppen bestehen. Dieser Umstand trägt mit zu dem Unbehagen (oder gar zu der Angst?) von Therapeuten gegenüber Gruppen bei. So entfalten gruppendynamische Prozesse oft eine Eigendynamik, die sich in Form von Subgruppenbildung, Konflikten oder auch offener Kritik gegenüber dem Gruppenleiter ausdrücken kann und die als wenig kontrollierbar erscheint. Die Vorliebe der Patienten, die - vor die Wahl gestellt, entweder Einzel- oder Gruppentherapie zu erhalten - sich in den meisten Fällen für erstere entscheiden, kommt dem Unbehagen der sie behandelnden Therapeuten entgegen. Es ist also davon auszugehen, dass die Entscheidung gegen Gruppen somit in stillem (weil unausgesprochenem) Einvernehmen von Therapeut und $\mathrm{Pa}$ tient getroffen wird. Schließlich mögen auch Zweifel an der Effektivität von Gruppen Therapeuten dazu veranlassen, diese im ambulanten Bereich erst gar nicht anzubieten.

Dabei spricht die Datenlage eindeutig für die Effektivität von Gruppentherapie! Bereits vor über 20 Jahren kamen Grawe, Donati und Bernauer [1994] zu einer Einschätzung, wonach «in einer großen Anzahl der von uns analysierten Therapiestudien ... die Therapien in Gruppen durchgeführt (wurden), auch wenn es überhaupt nicht um die Behandlung zwischenmenschlicher Probleme ging. Die Therapieeffekte waren in der Regel mindestens gleich gut wie bei der Behandlung im Einzelsetting». Die Effektivität gruppentherapeutischer Verfahren wurde zwischenzeitlich auch in mehr als 2 Dutzend Meta-Analysen untersucht. In diesen Studien

\section{KARGER}

() 2017 S. Karger GmbH, Freiburg 
ergaben sich dabei regelhaft Effektstärken in einer Größenordnung von 0,70 bis 0,90 (Interventions- vs. Kontrollgruppe)! Dabei belegen die Studien nicht nur in eindrucksvoller Weise die Effektivität von Gruppentherapie, sondern machen ebenfalls deutlich, dass die Gruppentherapie genauso effektiv ist wie die Einzeltherapie. Zwar gibt es Ausnahmen, die überwiegende Zahl der Studien belegt jedoch die Äquivalenz der beiden Formate. Lege artis durchgeführt, ist die Gruppentherapie also ebenso wirksam wie Einzeltherapie. Allerdings spricht einiges dafür, dass die Kombination der beiden Verfahren die besten Ergebnisse bedingt. Eine solche Kombination ließe sich auch in einer ambulanten Praxis leicht realisieren. Dabei wäre es durchaus plausibel, davon auszugehen, dass eine intelligente Kombination von Einzel- und Gruppentherapie die Effektivität therapeutischer Verfahren in signifikantem Maße erhöht.
Angesichts der eindeutigen Befundlage und der erwiesenermaßen vorliegenden ökonomischen Vorteile von Gruppentherapie verwundert es nicht, dass die Voraussetzungen dafür, Gruppentherapie im ambulanten Sektor anbieten zu können, durch das Versorgungsstärkungsgesetz verbessert werden sollen. So wird die Kombination von Einzel- und Gruppentherapie flexibilisiert, eine Antragstellung für die Durchführung von Kurzzeit-Therapiegruppen entfällt und Einzel- und Gruppentherapie können jeweils von unterschiedlichen Therapeuten angeboten werden, was die Organisation von Gruppen vereinfachen würde.

Gute Gründe, auch in der ambulanten Praxis (sehr) viel mehr Gruppen als bisher anzubieten, gab es schon lange. Mit dem Versorgungsstärkungsgesetzt fallen nun einige der Gründe weg, die bislang dagegen sprachen!

\section{Literatur}

Grawe K, Donati R, Bernauer F: Psychotherapie im Wandel: Von der Konfession zur Profession. Göttingen, Hogrefe, 1994, p 706.
Zielke M: Gruppenbehandlung in speziellen Settings: Verhaltensmedizinische Rehabilitation in der Psychosomatik; in Tschuschke V (Hrsg): Gruppenpsychotherapie. Von der Indikation bis zu Leitungstechniken. Stuttgart, Thieme, 2010, pp 164-168. 